burden that might be achieved by population shifts in these risk factors.

Methods The novel Stock of Health $(\mathrm{SoH})$ modelling approach integrates demographic and risk factor data from the Office for National Statistics and the Health Survey for England, and risk factor effects from the US Cardiovascular Lifetime Risk Pooling Project. The SoH approach estimates the latent "stock" of CHD-related health for each individual within the entire population of England and Wales (51 million). It then computes failure times for $\mathrm{CHD}$ mortality, allowing for multiplicative risk factor effects and competing mortality risks.

We first simulated CHD deaths for England and Wales over the period 2000-2010. We then extended the simulations to 2030, and compared "FEASIBLE" and "IDEAL" population intervention scenarios, targeted to systolic blood pressure (SBP) and cholesterol under two future trend assumptions: I) assuming mortality rates in 2010 remain stable through to 2030, or II) assuming CHD mortality continue falling.

Results The mean simulated failure times closely mirrored those actually observed(1993-2004: $m e n=72$ vs. 71 years; women=74 vs. 75 years). In 2030, about $81200 \mathrm{CHD}$ deaths are expected to occur under trend assumption I (+14\% compared to 2010, reflecting population ageing) and 47600 under trend assumption II (-18\% compared to 2010). SBP: For trend I, a FEASIBLE SBP reduction of $1 \mathrm{mmHg}$ and IDEAL reduction of $-5 \mathrm{mmHg}$ could result in approximately 77300 (+9\%, baseline: 2010$)$ and 64500 (-9\%, baseline: 2010$)$ deaths respectively in 2030. Assuming falling trends (II), about 45500 (-22\%, baseline: 2010) and 38300 (-34\%, baseline: 2010$)$ deaths would result in 2030 under the feasible and ideal scenarios respectively. Total cholesterol (TC): Under trend I, a FEASIBLE TC reduction of $0.1 \mathrm{mmol} / \mathrm{l}$ and IDEAL reduction of $0.5 \mathrm{mmol} / 1$ could result in about 74800 and 57000 deaths (5\% and $-20 \%)$ by 2030 . Assuming trend II, the resulting deaths would be approximately 43900 and 33400 ( $-25 \%$ and $-43 \%$, baseline: 2010$)$ respectively.

Conclusion Under both trend assumptions, the adoption of evidence-based dietary policies to reduce salt and saturated fat intake, resulting in population-wide decreases in blood pressure and cholesterol could result in substantial declines in forecasted CHD mortality.

\section{OP82 AN EVALUATION OF THE EFFECT OF THE NEW SCHOOL FOOD POLICY ON CHILDREN'S NUTRITIONAL INTAKE AND SOCIO-ECONOMIC CONSEQUENCES IN NORTH EAST ENGLAND}

doi:10.1136/jech-2012-201753.082

1,2S Spence, ' $\mathrm{J}$ Shen, ${ }^{1,2}$ J Delve, 'M White, 'L Vale, ${ }^{1,2} \mathrm{AJ}$ Adamson. 'Institute of Health and Society, Newcastle University, Newcastle upon Tyne, UK; ${ }^{2}$ Human Nutrition Research Centre, Newcastle University, Newcastle upon Tyne, UK

Background After 20 years with no regulation of school food and a plethora of evidence on the state of children's diets, new food and nutrient-based standards were re-introduced in 2006 to schools in England. As a major policy change receiving financial and legislative support from Government, our objectives were to evaluate the effect of this policy on children's nutritional intake, and its wider consequences including the added costs.

Methods A cross-sectional study was undertaken in primary $(\mathrm{n}=13)$ and middle $(\mathrm{n}=5)$ schools in North East England. Dietary, anthropometric and socio-economic data were collected from children aged 4-7y and 11-12y using identical quantitative methods pre, and post-implementation. In the 4-7y olds a four day food diary was completed using an observational method, the 11-12y olds completed $2 \times 3$ day food diaries followed by an interview. Economic analysis was conducted in the form of cost-consequence analysis, comparing the differences in costs with all of its possible consequences in a tabular format.
Results The effect of lunch type (school or packed) and year had a significant effect on total dietary intake in the 4-7y olds $(\mathrm{n}=1,017)$. Children having school lunches post-implementation had a slightly higher mean daily intake of energy $(93 \mathrm{kcals}$, $\mathrm{p}=0.004)$, but lower mean daily $\%$ energy from fat $(3 \%, \mathrm{p}<0.001)$ and saturated fat $(1 \%, \mathrm{p}<0 \cdot 001)$. Mean daily intakes of micronutrients such as vitamin $C$, and iron were higher in children consuming school lunches. In contrast, there was limited evidence of the effect of lunch type post-implementation on the total diet in $11-12 y$ olds $(n=883)$. The exception was \% energy from fat. In 1999-2000 children consuming a school lunch had a higher \% energy from fat than those consuming a packed lunch, post-implementation this difference was no longer apparent. The cost per school meal following implementation of the school food policy is higher than pre-implementation, ranging from $£ 29$ to $£ 55$ per child per year depending on how differences in prices of food pre and post-implementation were adjusted for inflation. Wider social consequences, for example, a reduction in socio-economic inequality, educational benefits and change in health behaviour were also set against the increased cost.

Conclusion These findings demonstrate that the introduction of the school food policy has the potential to have a positive impact not only on food eaten at school but also on children's total diet. Economic analysis highlights the trade-offs between significant improvement of nutrient intakes of children and the increased cost.

\section{OP83 DOES LUNCH TYPE HAVE AN IMPACT ON DIETARY QUALITY OVER THE WHOLE DAY IN ENGLISH PRIMARY SCHOOL CHILDREN?}

doi:10.1136/jech-2012-201753.083

CEL Evans, V Mandl. School of Food Science and Nutrition, University of Leeds, Leeds, UK

Background Nutrient standards were introduced by the Government to all English primary schools in 2008. By restricting some products and increasing the quantity and quality of others, the standards resulted in a healthier school meal profile. Despite attention to improve the quality of school meals, packed lunches have changed little in recent years and are not covered by similar regulations. There are few published studies that compare weight, body mass index (BMI), energy and nutrient intake over the whole day according to lunch type.

Methods A dietary assessment tool named CADET was used to assess the diet of 2355 children aged 6 to 8 years attending primary schools across England. Regression analysis which took into account the clustering of children within schools was used to report anthropometric, dietary and nutritional characteristics over one 24 hour period. Nutrient and anthropometric results from children having a packed lunch were compared with children having a school meal. Results were also compared to the reference nutrient intakes (RNIs) and the National Diet and Nutrition Survey (NDNS) in children.

Results No significant differences in weight, standardised BMI, or daily energy consumption were seen between children in the packed lunch group compared to the school meal group. However, there were nutritional differences, reported here as mean difference and $95 \%$ confidence interval $(95 \% \mathrm{CI})$. The packed lunch group consumed higher daily amounts of carbohydrates $(8.1 \mathrm{~g}, 95 \%$ CI 2.2 to 14.0g, $\mathrm{P}<0.01)$, sugar (9.8g, 95\% CI 5.4 to $14.3, \mathrm{P}<0.01)$ and sodium (92.1mg, 95\% CI 22 to $162 \mathrm{mg}, \mathrm{P}<0.01)$. Conversely, the school meal group consumed higher intakes of protein $(3.3 \mathrm{~g}, 95 \%$ CI 1.6 to $5 \mathrm{~g}$, $\mathrm{P}<0.01)$, fibre $(0.8 \mathrm{~g}, 95 \%$ CI 0.3 to $1.3 \mathrm{~g}, \mathrm{P}<0.01)$ and zinc $(0.2 \mathrm{mg}$, $95 \%$ CI 0.05 to $0.5 \mathrm{mg}, \mathrm{P}<0.01)$. Neither group met the recommended amounts for zinc, fibre or starch and indicated lower levels of sodium and higher levels of fat than the NDNS. Differences 
found in nutrient intake were independent of measures of child deprivation and ethnicity.

Conclusion Children taking a packed lunch to school have a less nutritious diet on average over the whole day in terms of sugar, sodium and fibre. A good quality lunch is therefore an important factor for overall dietary quality in children. Policies to encourage parents and children to include nutritious foods such as fruit, vegetables and low fat starchy foods are needed to narrow the gap between school meals and packed lunches.

\section{OP84 DO SUPERMARKET INTERVENTIONS IMPROVE FOOD ACCESS, FRUIT AND VEGETABLE INTAKE AND BMI? EVALUATION OF THE PHILADELPHIA FRESH FOOD FINANCING INITIATIVE}

doi:10.1136/jech-2012-201753.084

'E Flint, 'S Cummins, ${ }^{2}$ SA Matthews. 'School of Geography, Queen Mary University of London, London, UK; ${ }^{2}$ Department of Sociology, Pennsylvania State University, PA, USA

Background Structural interventions to improve access to healthy food in deprived communities are a current policy priority. In 2010, the Obama administration unveiled the Healthy Food Financing Initiative to promote interventions that expand access to nutritious foods. The initiative provided more than $\$ 400$ million in funding to bring grocery stores and health food retailers to deprived communities. However, no evidence for the effectiveness of such policy interventions exists. This paper is a first attempt to evaluate the effect of this intervention on perceptions of the food environment, fruit and vegetable intake and body mass index (BMI) in Philadelphia, USA.

Methods Data from the Philadelphia Neighbourhood Food Environment Study were used. This study utilised a prospective quasiexperimental design comparing baseline and follow up data in an 'intervention' community with a matched 'comparison' community. The intervention was the introduction of a large food supermarket in an underserved area. Outcomes were BMI, weekly fruit and vegetable consumption (Block FFQ), and perception of the neighbourhood food environment. Data were analysed using an intention-to-treat (ITT) and adopters vs non-adopters (ANA) approach. Difference-in-differences analyses (DID) assessed whether significantly different changes over time existed between intervention and comparison groups. Models were adjusted for age, sex, income, race, education, household composition and employment status.

Results In ITT and ANA analyses no significant difference-indifferences in mean BMI and mean weekly fruit and vegetable consumption were found. ITT analyses demonstrated a significant improvement in perceptions of the neighbourhood food environment in the intervention versus comparison neighbourhood (DID=1.71, $\mathrm{p}=0.001)$. Using ANA, a similar result was found for adopters, with those who used the grocery store as their primary shop (DID=1.57, $\mathrm{p}=0.05)$ and those who used it for any food shopping ( $\mathrm{DID}=2.34, \mathrm{p}<0.001$ ) having significantly better perceptions of the neighbourhood food environment compared to nonadopters. After adjustment, ITT analyses remained significant $(\mathrm{DID}=1.65, \mathrm{p}=0.002)$. For ANA, significant DID results were attenuated for primary adopters ( $\mathrm{DID}=1.30, \mathrm{p}=1.105$ ) but remained significant for those undertaking any food shopping (DID=2.18, $\mathrm{p}<0.001$ ).

Conclusion The introduction of a new grocery store had a positive effect on how local residents perceive the quality of their neighbourhood food environment. However, this did not have a significant effect on fruit and vegetable consumption or BMI. Improvement in perceptions of neighbourhood food environment quality did not translate into improved dietary behaviour or reductions in BMI.

\section{Population Based Studies: Mid Life and Older Age}

\section{OP85 DO GO0D HEALTH AND MATERIAL CIRCUMSTANCES PROTECT OLDER PEOPLE FROM THE INCREASE IN MORTALITY AFTER BEREAVEMENT?}

doi:10.1136/jech-2012-201753.085

'S M Shah, II M Carey, 'T Harris, 'S DeWilde, ${ }^{2} \mathrm{C}$ R Victor, 'D G Cook. 'Population Health Sciences and Education, St George's University of London, London, UK; ${ }^{2}$ School of Health Sciences and Social Care, Brunel University, Uxbridge, UK

Background Death of a spouse or partner is a common major life event for older people. The adverse health effects of bereavement are well recognised with an increased risk of death described in several populations. The impact of modifying factors, such as chronic disease and material circumstances, is less well understood. In this study, we use a large UK primary care database to examine the modifying and mediating effect of physical comorbidity and material socio-economic circumstances on the rise in mortality in the first year after bereavement.

Methods We identified 171,120 older (60 years and over) couples in a UK primary care database (THIN) based on a shared household identifier. The couples were followed up between 2005 and 2010 for an average of 4 years. 26,646 (15.5\%) couples experienced bereavement with mean follow up after bereavement of 2 years. The effect of bereavement on risk of death in the surviving partner was examined in a survival model adjusted for age, sex, comorbidity at baseline, material deprivation based on area of residence, season and smoking. Further analysis examined the effect of changes in comorbidity during follow up.

Results The fully adjusted hazard ratio (HR) for bereavement in the first year after bereavement was 1.25 (95\% CI: 1.18 to 1.33). Further adjustment for changes in comorbidity throughout follow up did not alter the hazard ratio for bereavement (HR 1.27, 95\% CI: 1.19 to 1.35). The effect of bereavement was not modified by age, gender or baseline comorbidity. The relative rise in mortality after bereavement was greatest in individuals with no significant chronic comorbidity throughout follow up (HR 1.50, 95\% CI: 1.28 to 1.77 ) and in more affluent couples $(P=0.035)$.

Conclusion We have confirmed the increased risk of mortality after bereavement and demonstrated its independence of pre-existing physician recorded chronic comorbidity and social status. Our analysis, taking account of changes in morbidity before and after bereavement, suggests that the rise in mortality after bereavement is not primarily mediated through new or worsening chronic physical disease. Furthermore, there was no evidence that pre-existing or continuing good health or affluence protect individuals. The results also suggest that, paradoxically, good health and high social status may accentuate the rise in mortality after bereavement. Our findings suggest that the rise in mortality after bereavement acts as a leveller, affording no protection to the affluent or healthy, and is best explained by an increase in sudden unexpected deaths.

\section{OP86 MORE RAPID DECLINE IN CHD INCIDENCE AMONG SCOTTISH TOWNS THAN AMONG ENGLISH TOWNS FROM THE 1980s TO THE 2000s}

doi:10.1136/jech-2012-201753.086

${ }^{1}$ E Cecil, 'SL Hardoon, 'SG Wannamethee, $2 \mathrm{PH}$ Whincup, 'RW Morris. 'Primary Care \& Population Health, UCL Medical School, London, UK; ${ }^{2}$ Population Health Sciences \& Education, St George's University of London, London, UK

Background The incidence of coronary heart disease (CHD) has declined in the UK since the late 1970s. However, regional differences in this decline are unknown. We investigate the change in 